\section{The new era of}

\section{spintronics}

Albert Fert, Jean-Marie George, Henri Jaffrès, Richard Mattana, and Pierre Seneor, Unité Mixte de Recherche CNRS-Thales (Associée à l'Université Paris-Sud), Orsay, 91404, France.

Spintronics, at the interface between magnetism and electron$S_{\mathrm{ics}}$, is a new field of research in considerable expansion [1]. The basic concept of spintronics is the manipulation of spin currents, in contrast to mainstream electronics in which the spin of the electron is ignored. Adding the spin degree of freedom provides new effects, new capabilities and new functionalities. Beyond today's applications to hard discs and memories, the potential of spintronics is very promising for new advances and an important impact on science and technology of the $21^{\text {st }}$ century.

The influence of the spin on the electronic transport properties of ferromagnetic materials has been known for a long time. The existence of spin polarized currents in ferromagnetic metals, first suggested by Mott, has been experimentally demonstrated and theoretically described about thirty years ago, mainly by European researchers (at Orsay, Strasbourg, Eindhoven). In metals like iron or cobalt, the "majority spin" and "minority spin" electrons carry currents that can be different by more than a factor of ten. This spin dependent conduction comes from the splitting of the "majority spin" and "minority spin" energy bands and also from the spin dependent cross section of some defects or impurities.

\section{Everybody has already a spintronic device on their desktop}

The first step on the road of the utilization of the spin degree of freedom was the discovery of the Giant Magnetoresistance of magnetic multilayers in 1988 [2]. A magnetic multilayer is a film composed of alternate ferromagnetic and nonmagnetic layers, $\mathrm{Fe}$ and $\mathrm{Cr}$ for example. The resistance of such a multilayer is lowest when the magnetic moments of ferromagnetic layers are aligned, and highest when they are antiparallel. As the relative change of resistance can be as high as $200 \%$, this effect has been called Giant Magnetoresistance (GMR). The condition required for GMR is a much better conduction through a ferromagnetic layer by the electrons of one of the spin directions, say the majority spin direction for example. When the magnetic moments of all the layers are aligned, half of the electrons are majority spin electrons in all the magnetic layers, and the short circuit effect by this high conduction channel results in a low resistance. In the antiparallel configuration, each electron is alternately a majority and a minority spin electron, the short circuit effect does not exist and the resistance is much higher. In specially designed multilayers, known as spin valves, the magnetic configuration can be switched between parallel and antiparallel configuration by a field of only a few Oersted, so that a large change of resistance can be induced by a very small field. The first spintronic devices have been based on spin valves. Today everybody has already a spintronic device on their desktop, since all modern computers use spin valves for the read heads of the hard disc. Because they can detect very small fields and very small magnetic bits, the spin valve-based read heads have led to an increase of the density of stored information by almost two orders of magnitude. It might however be hard to go beyond the $100 \mathrm{Gbit} / \mathrm{in}^{2}$ of today's prototypes with conventional spin valves. A further increase of read head sensitivity and information density in hard discs will be probably achieved with other spintronic devices, magnetic tunnel junctions [2] (see next paragraph), patterned multilayers for GMR in the non-conventional geometry where the current is perpendicular to the layers [4] or magnetic nanocontacts presenting a ballistic version of GMR known as BMR [5].

\section{Magnetic tunnel junctions and MRAM}

The magnetic tunnel junction (MTJ) [3] is the second type of spintronic device that will have soon important applications. A MTJ (Fig. 1a) is a structure in which two ferromagnetic layers (electrodes) are separated by a very thin insulating layer, commonly aluminum oxide. The electrons can tunnel through the insulating layer and, because the probability of tunneling from a ferromagnetic electrode depends on the spin direction, the resistance of the MTJ is different for the parallel and antiparallel orientations of the magnetic moments of the electrodes [3]. For electrodes of conventional ferromagnetic alloys, the relative change of resistance (Tunnel Magnetoresistance or TMR) can reach $70 \%$ at room temperature. MTJ of very small size, below the micron range, can be fabricated by lithographic techniques and an important application of these small size MTJ will be for a new type of computer memory, the MRAM (Magnetic Random Access Memory). As illustrated in the upper part of Fig.1, each junction can store one bit of data, say "0" for the parallel configuration of the magnetic moments of the electrodes, " 1 " for the antiparallel configuration. The sketch below is a schematic representation of a MRAM with MTJ connected together in a point contact array. The MRAM presently in development are expected to reach similar densities and access times as the current DRAM or SRAM, but their main advantage on these volatile semiconductor-based memories is that they retain data after the power is

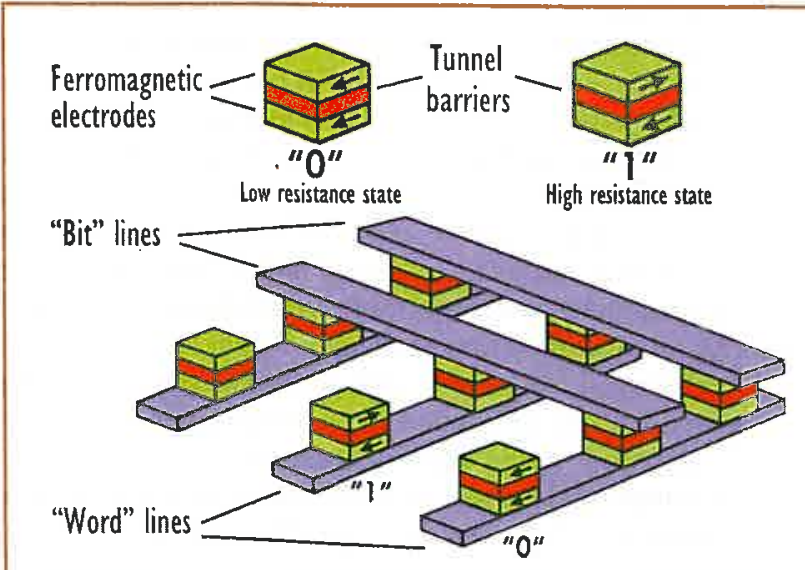

A Fig. 1 : Top: Memory cells of an MRAM (Magnetic Random Access Memory). Each cell is a submicronic MTJ and the states " 0 " and " 1 " of the cell corresponds respectively to the parallel and antiparallel configurations of the magnetic moments of electrodes of the MTJ (low and high resistance states). Central sketch: schematic of an MRAM constructed of MTJ connected together in a point contact array. Conducting wires, "bit lines" and "word lines" permit voltage measurements to read the stored information. They also enable manipulation of the magnetization of the elements (writing) by the magnetic field created by currents in the lines. The main advantage of MRAM over semiconductor-based DRAM or SRAM is their permanent character (for similar density and speed). 

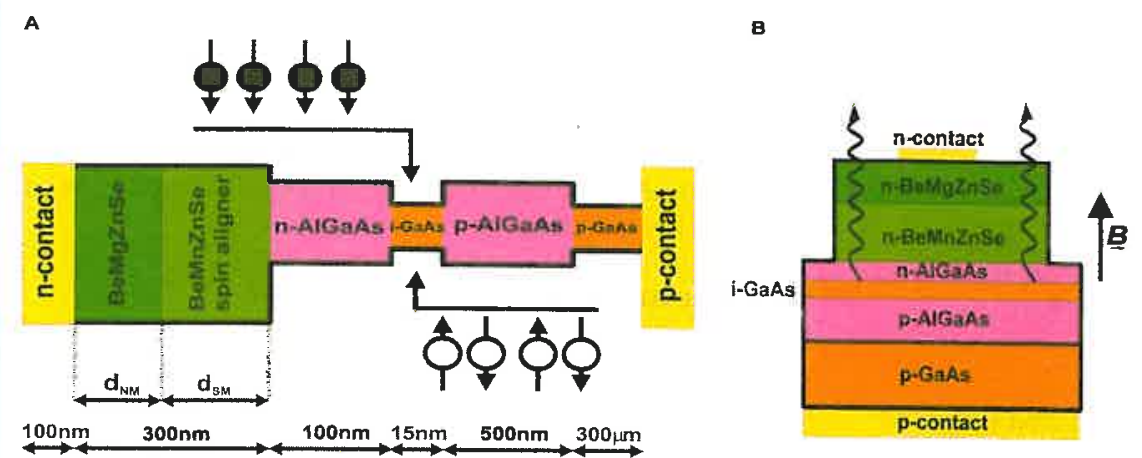

4Fig. 2: Spin Light Emitting Diade (SpinLED). A current of spin-polarized electrons is injected from the diluted magnetic semiconductor $\mathrm{Be}_{x} \mathrm{Mn}_{\mathrm{y}} \mathrm{Zn_{1-x } - \mathrm { y }}$ Se into a $\mathrm{GaAs} / \mathrm{GaAlAs}$ light-emitting diode $\mathrm{A}$ circularly polarized light is emitted from the recombination of the spin polarized electrons with non-polarized holes. An injection efficiency of $90 \%$ spin polarized current has been demonstrated by Fiederling et al. [10]. As Be $\mathrm{Mn}_{\mathrm{y}} \mathrm{Zn} \mathrm{n}_{1-\mathrm{x} y \mathrm{Se}}$ is paramagnetic, the spin polarization is obtained only in an applied field and at low temperature. turned off, possibly eliminating the long boot-up time when the computer is switched on. The MRAM have also an important advantage in terms of speed (a gain of 1000 for the write time) over the permanent memories of Flash type that are used today for mobile electronics. At least two commercial efforts-one by Motorola, the other by IBM and Infineon in Munich-plan to begin production of MRAM in 2004.

Though commercial products using MTJ are expected very soon, the physics of spin dependent tunneling is still far from being clearly understood. In the early research on the MTJ, it was thought that the spin polarization of the tunneling electrons and the TMR were simply reflecting the spin polarization of the electronic density of states at the Fermi level in the ferromagnetic clectrodes. It now turns out that the TMR also depends on the insulating material of the tunnel barrier and, more specifically, on the details of the electronic structure at the electrode/barrier interface. The present theories are not really quantitative yet and further theoretical developments are still needed for more reliable predictions. Another important challenge is the research of ferromagnetic materials providing higher spin polarizations than conventional metals like cobalt or iron. A few ferromagnets have been predicted to be half-metallic, that is to present a spin polarization of $100 \%$ at their Fermi level. A record TMR ratio of $1800 \%$, corresponding to a spin polarization of $95 \%$, has been found with electrodes of the manganite $\mathrm{La}_{2 / 3} \mathrm{Sr}_{1 / 3} \mathrm{MnO}_{3}$ (LSMO) [6]. However these results are obtained only at low temperature and LSMO cannot be considered for practical devices. Better candidates for room temperature applications seem to be magnetite $\left(\mathrm{Fe}_{3} \mathrm{O}_{4}\right)$ and several oxides of double perovskite structure. High TMR ratios might also be obtained with ferromagnetic insulating materials presenting a spin dependent gap and acting as spin filter for the tunneling electrons. Some interesting results have been recently obtained with EuS barriers, but at low temperature only.

\section{Spin transfer to reverse a magnetic moment without applying a magnetic field}

In a GMR or TMR device, switching the magnetic configuration changes the electrical current passing through the device. Magnetization reversal by spin transfer is an opposite effect: a spin polarized current is let into a device and the transfer of spin from the current switches the magnetic configuration of the device. This concept of magnetization reversal by spin transfer from a spin-polarized current has been introduced by Slonczewski [7] in 1995 and has been now confirmed by series of experiments (mainly on pillar-shaped multilayers). From the application point of view, reversing a magnetic moment by spin transfer without applying an external applied field can be of great interest to switch spintronic devices, MRAM for example. However the current density needed in the existing experiments is still relatively high, of the order of $10^{7} \mathrm{~A} / \mathrm{cm}^{2}$, and a better understanding of the spin transfer mechanisms seems necessary to obtain a significant reduction ot the current density. Another effect of the same type but probably requiring a smaller current density is the displacement of a domain wall by spin transfer from a spin-polarized current.

\section{Spintronics with semiconductors}

Whereas the metallic spin devices just described provide new ways to store and read information in hard discs, tapes or MRAM, semiconductor-based spintronics may offer a greater wealth of possibilities. Why is spintronics with semiconductors interesting? First, semiconductor-based spintronics could combine storage, detection, logic and communication capabilities on a single chip to produce a multifunctional device that could replace several components. For example, it could permit a better integration between MTJ and silicon-based electronics than in the present prototypes of MRAM. The

\section{A first interesting optical properties of the semicon-} ductors are also of particular interest property of the semiconductors to transform a magnetic information into an optical signal. Finally, because the manipulation of spins presents some advantages in term of for spintronics is speed and required power over the manipulation of charge in conventhe long electron tional electronics, some concepts of have been already proposed.

spin lifetime.

A first interesting property of the semiconductors for spintronics is the long electron spin lifetime. Fine time-resolved magneto-optical experiments have been developed at the University of Santa Barbara and have revealed spin lifetimes that can exceed $100 \mathrm{~ns}$ for electrons in bulk semiconductors at low temperature [8]. In heterostructures and quantum dots, nanosecond dynamics persist at room temperature [8]. In most of these experiments on spin dynamics, the spin polarization is created by optical excitation. However, for practical applications, it is highly desirable that the injection and detection of spin currents be electrical. The most direct way for spin injection would seem injecting from a classical ferromagnetic metal in a metal/semiconductor heterostructure but this raises difficult problems related to the difference in con- 
ductivity and spin relaxation time in metals and semiconductors. Although these problems are now better understood, this has slowed down the progress for spin injection from metals. On the other hand, this has boosted the research on diluted magnetic semiconductors (DMS) [9] that can be associated more easily with nonmagnetic semiconductors for spin injection. GaAs doped with Mn is an archetypal DMS which presents ferromagnetic properties up to $150 \mathrm{~K}$. Some other compounds are promising for room temperature ferromagnetism. We will describe two examples of spin injection from DMS in experiments respectively achieved at the University of Wurzburg [10] and in our team [12].
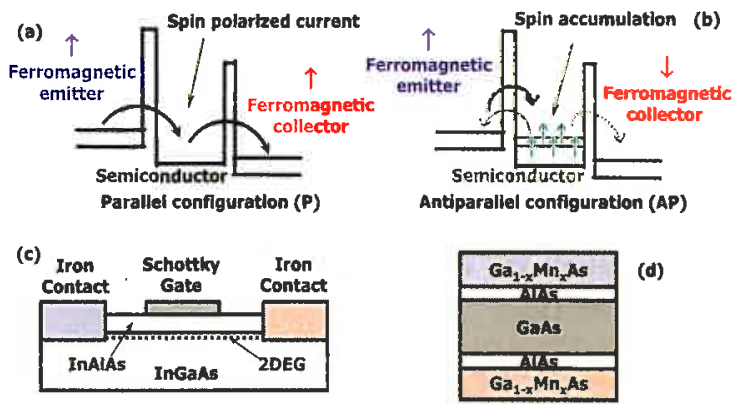

(e)
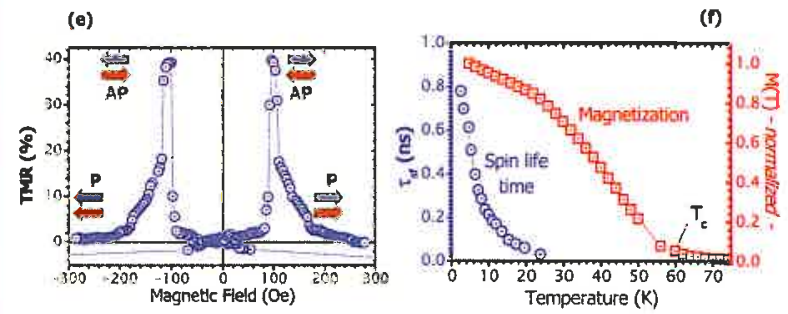

A Fig. 3: $(a, b)$ Sketches illustrating spin injection from a magnetic spin emitter (metal or semiconductor) into a semiconductor (2Delectron gas channel, Quantum Well, etc.) and spin detection by a magnetic collector (spin analyser). Injection and detection are through tunnel barriers. For a parallel $(P)$ configuration of magnetic moments in the emitter and collector (a), a spin-polarized current (spin up on the figure) is injected and transmitted to the collector. For an antiparallel (AP) configuration (b), spin up electrons are injected and accumulated in the semiconductor (accumulation due to the poor transmission to the collector) and rejected in major part by the spin up accumulation into the emitter. The condition for strong accumulation and large difference between the resistances of the $P$ and AP configurations is that the spin lifetime in the semiconductor be longer than the time spent by the particle in the semiconductor. (c) Lateral heterostructure with metallic spin emitter and spin analyser for spin injection into a 2D Electron Gas (DEG). With a Schottky gate to rotate the spin polarization by the Rashba effect, this device is the Spin Field Effect Transistor proposed by Datta and Das [11]. (d) Vertical heterostructure for injection of spin-polarized holes into a GaAs Quantum Well (QW) in the experiments of Mattana et al. [12]. Emitter and analyser are layers of the ferromagnetic semiconductor $\mathrm{Ga}_{1-x} \mathrm{Mn} \mathrm{n}_{\mathrm{x}} \mathrm{As}$. (e)

Magnetoresistance of a heterostructure of the type of (d) [12]. (f) Temperature dependence of the s.pin life time in a GaAs QW from magnetoresistnace measurements on a heterostructures of the type of (d).
In the heterostructure of Fig.2, spin-polarized electrons are injected from a paramagnetic DMS $\left(\mathrm{Be}_{\mathrm{x}} \mathrm{Mn}_{\mathrm{y}} \mathrm{Zn}_{1-x-y} \mathrm{Se}\right)$ into a GaAs/AlGaAs light emitting diode (LED), which leads to emission of circularly polarized light. An injection efficiency of $90 \%$ spin polarized current has been demonstrated with this structure [10]. Similar experiments but with smaller polarization have also been performed with spin injection from ferromagnetic GaMnAs and from metals.

Fig. 3 illustrates the physics of devices where both injection of spins into the semiconductor and detection of the spin information are electrical. The ideal situation is when the spin lifetime is much longer than the time spent by the carriers in the semiconductor. As illustrated in Fig.1(a,b), a spin-polarized current is then easily transmitted in the parallel configuration of emitter and collector, whereas the antiparallel one leads to spin accumulation and current blockade. In the Datta and Das [11] spin transistor of Fig.3(c), a gate voltage can rotate the spins to switch the device between the two regimes. Fig.3(d) represents an example of vertical version of such an emitter/semiconductor/analyser structure, and, in Fig.3(e), we show the magnetoresistance resulting from the coherent transmission of the spin information in this device [12]. The temperature dependence of the spin lifetime in the GaAs QW derived from this magnetoresistance is shown in Fig.3(f).

The two examples of Fig. 2 and 3 show it is now possible to inject spins electrically into a semiconductor heterostructure and to transform the spin information into an optical (Fig.2) or electrical signal (Fig.3). This paves the way for more advanced spintronic devices in which it will be possible to manipulate the spin polarization between emitter and analyser.

In just a dozen of years, we have seen spintronics increasing considerably the capacity of hard disks and now getting ready to enter the RAM of computers. In the next decade, spintronics with semiconductors has the potential to gain an important place in the microelectronics industry. Another perspective, at longer term and out of the scope of this paper, is the exploitation of the truly quantum-mechanical nature of spin and the long spincoherence time in confined geometry for quantum computing in an even more revolutionary application.

\section{References.}

[1] For a recent detailed review, see S. A. Wolf et al., Science 294, 1488, 2001.

[2] M. Baibich et al., Phys. Rev. Lett.61, 2472, 1988; Binasch et al., Phys. Rev. B39, 4828, 1989.

[3] M. Jullière, Phys. Lett. 54A, 225, 1975; J. S. Moodera et al., Phys. Rev. Lett.74, 3273, 1995.

[4] J. Bass and W. P. Pratt, J. Mag. Mag. Mat. 200, 274, 1999.

[5] N. Garcia et al, Phys. Rev. Lett. 82, 2923, 1999.

[6] M. Bowen et al., Appl. Phys. Lett. 82, 233, 2003.

[7] J. Slonczewski, J. Mag. Mag. Mat. 159, 1, 1996.

[8] J. M. Kikkawa et al, Science 277, 1284, 1997; Phys. Rev. Lett. 80, 4313, 1998.

[9] T. Dietl et al., Science 287, 1019, 2000.

[10] R. Fiederling et al., Nature 402, 787, 1999.

[11] S. Datta and B. Das, Appl. Phys. Lett. 56, 665, 1990.

[12] R. Mattana et al., Phys. Rev. Lett. 90, 166601, 2003. 